\title{
Metal Exposure and Associated Health Risk to Human Beings by Street Dust in a Heavily Industrialized City of Hunan Province, Central China
}

\author{
Guangyi Sun ${ }^{1,2}$, Zhonggen $\mathrm{Li}^{1, *}$, Ting Liu ${ }^{1,2,3}$, Ji Chen ${ }^{1,4}$, Tingting $\mathrm{Wu}^{1,5}$ and Xinbin Feng ${ }^{1, *}$ \\ 1 State Key Laboratory of Environmental Geochemistry, Institute of Geochemistry, Chinese Academy of \\ Sciences, Guiyang 550081, China; sunguangyi@mail.gyig.ac.cn (G.S.); liuting@ihb.ac.cn (T.L.); \\ jigerchen@163.com (J.C.); wutingtingcc@163.com (T.W.) \\ 2 University of Chinese Academy of Sciences, Beijing 100190, China \\ 3 Institute of Hydrobiology, Chinese Academy of Sciences, Wuhan 430072, China \\ 4 Guizhou Provincial Laboratory for Mountainous Environment, Guizhou Normal University, \\ Guiyang 550001, China \\ 5 Key Laboratory of Karst Environment and Geohazard Prevention, Guizhou University, \\ Guiyang 550003, China \\ * Correspondence: lizhonggen@vip.skleg.cn (Z.L.); fengxinbin@vip.skleg.cn (X.F.); \\ Tel.: +86-13608517028 (Z.L.); Fax: +86-851-8589-1334 (Z.L.)
}

Academic Editor: Susanne Charlesworth

Received: 15 December 2016; Accepted: 22 February 2017; Published: 3 March 2017

\begin{abstract}
Fifty-five urban street dust samples were collected from Zhuzhou, an industrial city in central China and analyzed for a range of toxic elements. Potential carcinogenic and non-carcinogenic health effects on children and adults due to exposure to street dust were assessed. Concerning the two subgroups, the child cohort is confronted with considerably greater health risks than adults. According to the Hazard Quotient (HQ) method, ingestion of dust particles poses primary risk to children and adults, followed by dermal contact and inhalation for all of the metals investigated except $\mathrm{Hg}$, for which inhalation of its elemental vapor constitute a slightly higher risk than ingestion. For children, $\mathrm{Pb}, \mathrm{As}, \mathrm{Cd}, \mathrm{Cr}, \mathrm{Hg}$ and $\mathrm{Sb}$ exposure were deemed as the highest contributors to non-cancer health risks, while As and $\mathrm{Cr}$ represent an enhanced cancer risk for children. For adults, risk indicator values for both cancer and non-cancer effects obtained were within the safety threshold. In a comparison with other locations within and outside mainland China, exposure to arsenic is prominent for the population of Zhuzhou, indicating more attention and preventive actions should been taken.
\end{abstract}

Keywords: street dust; health risk; cancer; arsenic; metals

\section{Introduction}

Street dust, a special type of environmental medium with a complex composition in urban regions, could potentially cause adverse effects on exposed populations. Metal contamination character and source identification are the main concern of most previous literature on street dust [1-6]. On the basis of toxic metal contents solely, it is difficult to produce a quantitative health assessment. Although some metals are considerably enriched in street dust, both cancer and non-cancer risk normally falls below the acceptable level by the corresponding regulatory authorities [7]. Researches both from epidemiological and toxicological studies have showed that metals and metalloids can accumulate in fatty tissues, affecting the functions of organs and disrupting the nervous system or endocrinal system; or interact directly with DNA to cause mutations [8-13]. Nonetheless, in heavy impacted municipalities, there is an urgent need to assess the health risk associated with toxic metal exposure 
for the residents' health. Human health risk assessment has proved to be a useful tool to pinpoint the severity of various toxic metals [14-19]. The health risk, a method combining metal pollution and human health, is developed from a qualitative or quantitative estimate of the likelihood that any of the hazards associated with the agent of concern will be realized in exposed people [20].

The objectives of the present study were to estimate health risk for children and adults due to toxic metal exposure to street dust according to Hazard Indexes (HI) and cancer risk assessment in Zhuzhou, a heavily industrialized city in China, and put the result into a broader perspective of similar studies in China and elsewhere.

\section{Materials and Methods}

\subsection{Study Area}

Zhuzhou, the second largest city of Hunan Province in central China, is located in the middle reaches of the Xiang River watershed (Figure 1) and about $50 \mathrm{~km}$ south of Changsha, the provincial capital. The city developed rapidly from a town in the 1950s to a large city, with a population of 1.002 million and a built-up area of $105 \mathrm{~km}^{2}$ by the end of 2009. Zhuzhou has a typical north subtropical monsoon climate, with prevailing NNW winds, except during summer (June-August) with SE winds. Its annual average temperature is $17.6^{\circ} \mathrm{C}$ with plenty of precipitation, mainly during March to August, with an annual rainfall of $1409 \mathrm{~mm}$. The topography mostly consists of alluvial plains and gentle hills at an elevation of 50-200 m above sea level. Acidic ferro soils of krasnozem type are here predominant as elsewhere in southern China [21].

Zhuzhou is a major base of heavy industries in Hunan Province. Zhuzhou Electrical Railway Engine Corporation, Zhuzhou Hard Alloy Group, China Southern Power Equipment Corporation and Zhuzhou Smelting Group are all backbone enterprises of the city, predominantly established during the era of China's first and second "Five-year Plan" (1953-1962). The city's industrial output is characterized by production foremost in the categories of metallurgy, machinery, chemistry and building materials [21].

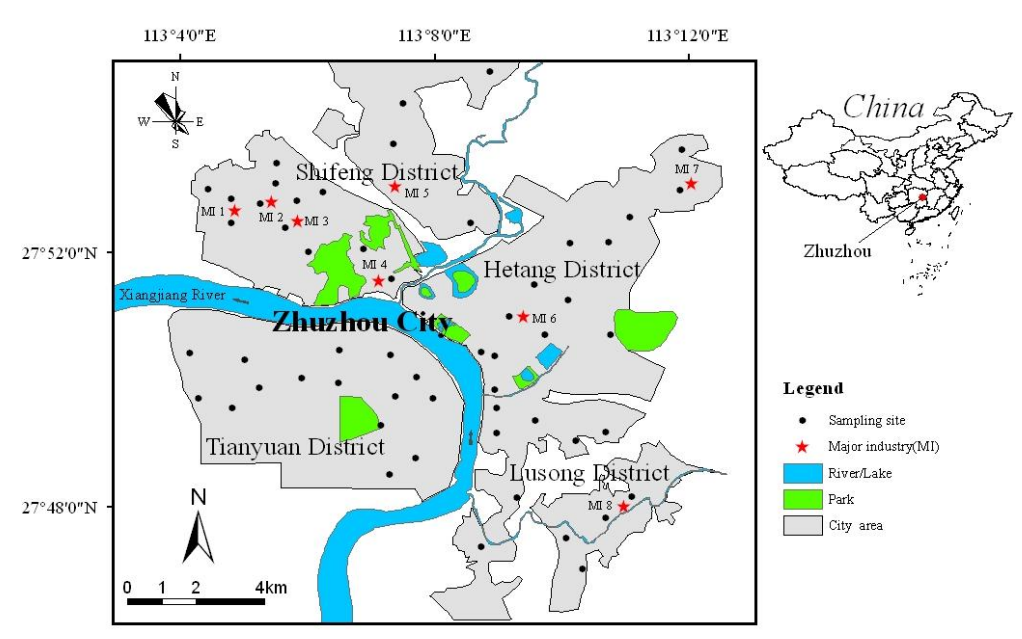

Figure 1. Sketch map showing the study area with sampling locations of street dust and the major industries in Zhuzhou city.

\subsection{Data Sources}

The street dust samples were collected in winter of 2010. A total of 55 samples were obtained across the city, as shown in Figure 1. The levels, spatial distributions and possible sources of heavy metals ( $\mathrm{Ag}, \mathrm{As}, \mathrm{Cd}, \mathrm{Co}, \mathrm{Cr}, \mathrm{Cu}, \mathrm{Hg}, \mathrm{Mo}, \mathrm{Ni}, \mathrm{Pb}, \mathrm{Sb}, \mathrm{Zn}$ ) in street dust were reported by our previous publication ( $\mathrm{Li}$ et al. [21]). The data showed that the $\mathrm{Zn} / \mathrm{Pb}$ smelting activities located in the northwest part of city are the main source of $\mathrm{Ag}, \mathrm{As}, \mathrm{Cd}, \mathrm{Cu}, \mathrm{Hg} \mathrm{Pb}, \mathrm{Sb}$ and $\mathrm{Zn}$, while a hard alloy plant in the 
central city responsible for the Mo enrichment, nevertheless $\mathrm{Co}, \mathrm{Cr}$ and $\mathrm{Ni}$ are mainly ascribed to the natural sources.

\subsection{Risk Assessment Method}

Human exposure to trace elements in street dust can induce health risks via three main paths: (a) direct ingestion of substrate particles $\left(D_{\text {ing }}\right)$; (b) inhalation of suspended particles through mouth and nose $\left(D_{\text {inh }}\right)$; and (c) dermal absorption of potentially harmful elements in particles adhered to exposed skin $\left(D_{\text {dermal }}\right)$. The dose received through each of the three pathways was calculated using Equations (1)-(3), which were adopted from U.S. Environmental Protection Agency [22,23] and Zheng et al. $[15,16]$. For $\mathrm{Hg}$, exposure via inhalation of its elemental vapor is viable following Equation (4). For carcinogens, lifetime average daily dose (LADD) is used for assessment of cancer risk. LADD is calculated as a weighted average for each exposure route as given in Equation (5):

$$
\begin{gathered}
D_{\text {ing }}=C \times \frac{I n g R \times E F \times E D}{B W \times A T} \times 10^{-6} \\
D_{\text {inh }}=C \times \frac{I n h R \times E F \times E D}{P E F \times B W \times A T} \\
D_{\text {dermal }}=C \times \frac{S L \times S A \times A B S \times E F \times E D}{B W \times A T} \times 10^{-6} \\
D_{\text {vapour }}=C \times \frac{I n h R \times E F \times E D}{V F \times B W \times A T} \\
L A D D=C \times \frac{C R \times E F \times E D}{P E F \times B W \times A T}
\end{gathered}
$$

The formulae acronyms used in Equations (1)-(5) are explained in Table 1. The reference values used in the analysis were retrieved from U.S. EPA [24], Van den Berg [19], Naturvårdsverket [25] and De Miguel [24]. Site specific biometric factors were assessed from literature recommendations [19,24].

$\mathrm{C}$ (exposure-point concentration, $\mathrm{mg} \cdot \mathrm{kg}^{-1}$ ) in Equations (1)-(5), combined with the values of exposure factors shown above, are considered to yield an estimate of the "reasonable maximum exposure" [22], which is the upper limit of the 95\% confidence interval for mean (UCL, upper confidence limit) [23]. Considering that the concentration of each trace element investigated followed log-normal distribution among samples, 95\% UCL was calculated using the statistical software SPSS 15.0 for Windows (SPSS Inc., Chicago, IL, USA).

The potential carcinogenic and non-carcinogenic risks for human being were calculated using Equations (6) and (7) [26].

$$
\begin{aligned}
& \text { Carcinogenic Risk }=L A D D \times S F \\
& \text { Hazard quotient }(\mathrm{HQ})=D_{i} / R f D
\end{aligned}
$$

For carcinogens, the carcinogenic risk is multiplied by the corresponding slope factor (SF) to produce a level. The acceptable or tolerable risk for regulatory purposes is in the range of $1 \times 10^{-6}-1 \times 10^{-4}$ [19]. RfD is the reference dose $\left(\mathrm{mg}^{-\mathrm{kg}^{-1}}\right.$.day $\left.{ }^{-1}\right)$ and $\mathrm{D}_{\mathrm{i}}$ is the daily exposure amounts of the selected metal ( $\left.\mathrm{mg} \cdot \mathrm{kg}^{-1} \cdot \mathrm{day}^{-1}\right)$. Hazard Quotient (HQ) for non-carcinogenic effects was applied to each exposure pathway in the analysis. The approach assumes that simultaneous sub-threshold exposures to toxic metals could result in adverse health effects and the magnitude of the adverse effect will be proportional to the sum of the ratios of the sub-threshold exposures to acceptable exposures [22]. Hazard index (HI) is equal to the sum of HQ. If the value of $\mathrm{HI}$ is less than one, it is assumed that there is no significant risk of non-carcinogenic effects. If $\mathrm{HI}$ exceeds one, then there is a chance that non-carcinogenic effects occur, with a probability which tends to increase as the value of $\mathrm{HI}$ increases [24]. For a comparison, relative toxicity values (RfD and SF) from this study and from the literature are summarized in Table 2. 
Table 1. Description of parameters and the default values used in does and health risk assessment.

\begin{tabular}{|c|c|c|c|}
\hline Symbol & Meanings & Default Value & Data Source, Year [Reference] \\
\hline C & Value of elements in street dust & $95 \%$ UCL & This study \\
\hline $\operatorname{IngR}$ & Ingestion rate & $200 \mathrm{mg} \cdot$ day $^{-1}$ (child); $100 \mathrm{mg} \cdot$ day $^{-1}$ (adult) & U.S. EPA, 2001 [24] \\
\hline $\operatorname{Inh} R$ & Inhalation rate & $7.6 \mathrm{~m}^{3} \cdot$ day $^{-1}$ (child); $20 \mathrm{~m}^{3} \cdot$ day $^{-1}$ (adult) & Van den Berg, $1995[27]$ \\
\hline$E F$ & Exposure frequency (site specific) & 180 day $\cdot$ year $^{-1}$ & Ferreira-Baptista and De Miguel, 2005 [14] \\
\hline$E D$ & Exposure duration & 6 year (child); 24 year (adult) & U.S. EPA, 1996, $2001[23,24]$ \\
\hline$S A$ & Skin area exposed & $1150 \mathrm{~cm}^{2}$ (child); $2145 \mathrm{~cm}^{2}$ (adult) & Wang et al. 2008 [28] \\
\hline SL & Skin adherence factor & $0.2 \mathrm{mg} \cdot \mathrm{cm}^{-2} \cdot$ day $^{-1}$ (child); $0.07 \mathrm{mg} \cdot \mathrm{cm}^{-2} \cdot$ day $^{-1}$ (adult) & U.S. EPA, 1996, 2001 [23,24] \\
\hline$A B S$ & Dermal absorption factor & 0.03 (for As); 0.001 (for other elements) & U.S. DOE, 2004 [20] \\
\hline PEF & Particle emission factor & $1.36 \times 10^{9} \mathrm{~m}^{3} \cdot \mathrm{kg}^{-1}$ & U.S. EPA, 2001 [24] \\
\hline$V F$ & Volatility factor & $32,675.6 \mathrm{~m}^{3} \cdot \mathrm{kg}^{-1}$ (elemental mercury vapor) & U.S. EPA, $2001[24]$ \\
\hline$B W$ & Body weight & $15.4 \mathrm{~kg}$ (child); $56 \mathrm{~kg}$ (adult) & U.S. EPA, 1996, 2001;Wang et al. $2005[23,24,29]$ \\
\hline$A T$ & Average time & $E D \times 365$ (for non-carcinogens) $E D \times 365$ (for carcinogens) & U.S. EPA, 1989 [22] \\
\hline$C R$ & Contact (absorption) rate & $\begin{array}{c}\text { Ingestion: }[C R=\operatorname{IngR}] \text {; Inhalation: }[C R=\operatorname{Inh} R] \\
\text { Dermal: }[C R=S A \times S L \times A B S]\end{array}$ & U.S. EPA, 1996, 2001 [23,24] \\
\hline
\end{tabular}

Table 2. Relative toxicity values used in this study [14-16,23].

\begin{tabular}{|c|c|c|c|c|c|c|c|c|c|c|c|c|}
\hline & Ag & As & $\mathrm{Cd}$ & Co & $\mathrm{Cr}$ & $\mathrm{Cu}$ & $\mathrm{Hg}$ & Mo & $\mathrm{Ni}$ & $\mathbf{P b}$ & $\mathrm{Sb}$ & $\mathrm{Zn}$ \\
\hline Oral RfD & $5.00 \times 10^{-3}$ & $3.00 \times 10^{-4}$ & $1.00 \times 10^{-3}$ & $2.00 \times 10^{-2}$ & $3.00 \times 10^{-3}$ & $4.00 \times 10^{-2}$ & $3.00 \times 10^{-4}$ & $5.00 \times 10^{-3}$ & $2.00 \times 10^{-2}$ & $3.50 \times 10^{-3}$ & $4.00 \times 10^{-4}$ & $3.00 \times 10^{-1}$ \\
\hline $\mathrm{D} \times 10 \mathrm{rmal} . \mathrm{RfD}$ & $9.00 \times 10^{-4}$ & $1.23 \times 10^{-4}$ & $1.00 \times 10^{-5}$ & $1.60 \times 10^{-2}$ & $6.00 \times 10^{-5}$ & $1.20 \times 10^{-2}$ & $2.10 \times 10^{-5}$ & $1.90 \times 10^{-3}$ & $5.40 \times 10^{-3}$ & $5.25 \times 10^{-4}$ & $8.00 \times 10^{-6}$ & $6.00 \times 10^{-2}$ \\
\hline Inhal. RfD & $5.00 \times 10^{-3}$ & $3.00 \times 10^{-4}$ & $1.00 \times 10^{-3}$ & $5.71 \times 10^{-6}$ & $2.86 \times 10^{-5}$ & $4.02 \times 10^{-2}$ & $2.90 \times 10^{-4}$ & $4.95 \times 10^{-3}$ & $2.06 \times 10^{-2}$ & $3.52 \times 10^{-3}$ & $4.00 \times 10^{-4}$ & $3.00 \times 10^{-1}$ \\
\hline Vapour RfD & & & & & & & $8.57 \times 10^{-5}$ & & & & & \\
\hline Oral SF & & $1.50 \times 10$ & & & & & & & & & & \\
\hline $\mathrm{D} \times 10 \mathrm{rmal} \mathrm{SF}$ & & $3.66 \times 10$ & & & & & & & & & & \\
\hline Inhal. SF & & $1.51 \times 10$ & $6.30 \times 10$ & $9.80 \times 10$ & $4.20 \times 10^{1}$ & & & & $8.40 \times 10^{-1}$ & & & \\
\hline
\end{tabular}




\section{Results and Discussion}

\subsection{Trace Elements in the Street Dust}

Descriptive statistical results of trace element concentrations investigated in Zhuzhou city, as well as their background value in surface soils of Hunan province [30], are summarized in Table 3. In particular, the average concentration of $\mathrm{Zn}$ and $\mathrm{Pb}$ is enhanced at $2379 \mathrm{mg} \cdot \mathrm{kg}^{-1}$ and $956 \mathrm{mg} \cdot \mathrm{kg}^{-1}$, respectively. The arithmetic mean concentration for elements followed in the order of $\mathrm{Zn}>\mathrm{Pb}>\mathrm{Cu}$ $>\mathrm{Cr}>\mathrm{As}>\mathrm{Cd}>\mathrm{Ni}>\mathrm{Sb}>\mathrm{Co}>\mathrm{Mo}>\mathrm{Ag}>\mathrm{Hg}$.

Table 3. Trace elements concentrations in street dust collected form Zhuzhou city [21] $\left(\mathrm{N}=55, \mathrm{mg} \cdot \mathrm{kg}^{-1}\right)$.

\begin{tabular}{cccccccccc}
\hline Element & Median & Mean & SD & Min & Max & 95\% UCL & Skewness & Reference Value & EF \\
\hline $\mathrm{Ag}$ & 1.17 & 2.49 & 4.56 & 0.46 & 28.7 & 3.72 & 4.49 & 0.108 & 10.83 \\
$\mathrm{As}$ & 41.8 & 87.8 & 182.9 & 15.4 & 1194.1 & 137.2 & 5.05 & 15.7 & 2.7 \\
$\mathrm{Cd}$ & 10.3 & 41.4 & 117.3 & 2.2 & 691 & 73.1 & 4.71 & 0.126 & 81.75 \\
$\mathrm{Co}$ & 13 & 15 & 11 & 8 & 84 & 18 & 4.85 & 14.6 & 0.86 \\
$\mathrm{Cr}$ & 115 & 124.6 & 54.1 & 59.5 & 302 & 139 & 1.78 & 71.4 & 1.61 \\
$\mathrm{Cu}$ & 97.6 & 139 & 148.3 & 39.2 & 1020 & 179 & 4.44 & 27.3 & 3.58 \\
$\mathrm{Hg}$ & 0.21 & 0.92 & 2.7 & 0.08 & 14.6 & 1.65 & 4.69 & 0.116 & 1.77 \\
$\mathrm{Mo}$ & 3.2 & 6.4 & 12.4 & 1.3 & 90.1 & 9.7 & 6.02 & 1.4 & 2.25 \\
$\mathrm{Ni}$ & 35 & 40 & 16 & 20 & 105 & 45 & 1.95 & 31.9 & 1.1 \\
$\mathrm{~Pb}$ & 254 & 956 & 2815 & 96 & 17,578 & 1717 & 5.03 & 29.7 & 8.54 \\
$\mathrm{Sb}$ & 9.8 & 15.8 & 21.7 & 3.7 & 115.4 & 21.7 & 3.81 & 1.87 & 5.24 \\
$\mathrm{Zn}$ & 1140 & 2379 & 5145 & 317 & 35,400 & 3770 & 5.46 & 94.4 & 12.08 \\
\hline
\end{tabular}

SD: Std. Deviation; Min: Minimum; Max: Maximum; Reference value in the soil of Hunan province, (CNEMC, 1990) [30]; EF, enrichment factor $=$ median/reference value .

The concentration distribution in samples for all of the studied elements display a positive skewness $(>1)$ and can be approximated as a log-normal one. Furthermore, enrichment factors (EFs) for all the elements except for Co are greater than unity signifying anthropogenic influences. Among the elements, the magnitude of EF peaking for Cd (81.75) but is also considerably high $(>10)$ for some adjacent elements in the periodical table, namely $\mathrm{Zn}$ and $\mathrm{Ag}$.

\subsection{Non-Carcinogenic Risk of Metals}

For non-carcinogenic health effects among children and adults, the calculated overall risk and the corresponding contribution for each exposure pathway are summarized in Table 4 . In the case of children, the rank for exposure risk among the three routes was for all elements ingestion $>$ dermal contact > inhalation. $\mathrm{Hg}$ is an exception, for which inhalation of $\mathrm{Hg}$ vapor is the primary cause of exposure and not by particulate matter [13]. This result also applies to adults. Similar results were obtained by Ferreira-Baptista and DeMiguel [14], and Zheng et al. [21,22].

In general, children exposure non-cancer risks of toxic metals were nearly one order of magnitude higher than the values of adult. So, children are confronted by greater harmful health risks due to the street dust than other population groups. This is similar to other reports [7].

HIs for metals to children and adult decrease in the order of $\mathrm{Pb}>\mathrm{As}>\mathrm{Cd}>\mathrm{Sb}>\mathrm{Cr}>\mathrm{Hg}>$ $\mathrm{Zn}>\mathrm{Cu}>\mathrm{Ni}>\mathrm{Mo}>\mathrm{Co}>\mathrm{Ag}$ and $\mathrm{Pb}>\mathrm{As}>\mathrm{Hg}>\mathrm{Cd}>\mathrm{Sb}>\mathrm{Cr}>\mathrm{Zn}>\mathrm{Cu}>\mathrm{Ni}>\mathrm{Mo}>\mathrm{Co}>\mathrm{Ag}$, respectively, The above showed that $\mathrm{Pb} / \mathrm{Zn}$ smelting may be the most important factor raising risk to human health, due to $\mathrm{Pb}$ mainly related to $\mathrm{Pb} / \mathrm{Zn}$ smelting. Except for $\mathrm{Pb}$ and $\mathrm{As}, \mathrm{HIs}$ for other metals due to street dust exposure in this study are lower than the safety limit of unity, indicating less non-cancer risks from these elements for children and adult. 
Table 4. Daily exposure amounts and hazard quotient of metals in street dust to adult and child through three routes.

\begin{tabular}{|c|c|c|c|c|c|c|c|c|c|c|c|c|}
\hline $\mathrm{mg} \cdot \mathrm{kg}^{-1} \cdot \mathrm{day}^{-1}$ & $\mathrm{Ag}$ & As & $\mathrm{Cd}$ & Co & $\mathrm{Cr}$ & $\mathrm{Cu}$ & $\mathrm{Hg}$ & Mo & $\mathrm{Ni}$ & $\mathrm{Pb}$ & $\mathrm{Sb}$ & $\mathrm{Zn}$ \\
\hline \multicolumn{13}{|l|}{ Adult } \\
\hline Ding & $3.28 \times 10^{-6}$ & $1.21 \times 10^{-4}$ & $6.44 \times 10^{-5}$ & $1.59 \times 10^{-5}$ & $1.22 \times 10^{-4}$ & $1.58 \times 10^{-4}$ & $1.45 \times 10^{-6}$ & $8.54 \times 10^{-6}$ & $3.96 \times 10^{-5}$ & $1.51 \times 10^{-3}$ & $1.91 \times 10^{-5}$ & $3.32 \times 10^{-3}$ \\
\hline $\mathrm{Dd} \times 10 \mathrm{rmal}$ & $4.92 \times 10^{-9}$ & $5.44 \times 10^{-6}$ & $9.67 \times 10^{-8}$ & $2.38 \times 10^{-8}$ & $1.84 \times 10^{-7}$ & $2.37 \times 10^{-7}$ & $2.18 \times 10^{-9}$ & $1.28 \times 10^{-8}$ & $5.95 \times 10^{-8}$ & $2.27 \times 10^{-6}$ & $2.87 \times 10^{-8}$ & $4.98 \times 10^{-6}$ \\
\hline Dinh & $4.82 \times 10^{-10}$ & $1.78 \times 10^{-8}$ & $9.47 \times 10^{-9}$ & $2.33 \times 10^{-9}$ & $1.80 \times 10^{-8}$ & $2.32 \times 10^{-8}$ & $2.14 \times 10^{-10}$ & $1.26 \times 10^{-9}$ & $5.83 \times 10^{-9}$ & $2.22 \times 10^{-7}$ & $2.81 \times 10^{-9}$ & $4.88 \times 10^{-7}$ \\
\hline Dvapour & & & & & & & $8.89 \times 10^{-6}$ & & & & & \\
\hline HQing & $6.55 \times 10^{-4}$ & $4.03 \times 10^{-1}$ & $6.44 \times 10^{-2}$ & $7.93 \times 10^{-4}$ & $4.08 \times 10^{-2}$ & $3.94 \times 10^{-3}$ & $4.84 \times 10^{-3}$ & $1.71 \times 10^{-3}$ & $1.98 \times 10^{-3}$ & $4.32 \times 10^{-1}$ & $4.78 \times 10^{-2}$ & $1.11 \times 10^{-2}$ \\
\hline $\mathrm{HQd} \times 10 \mathrm{rmal}$ & $5.47 \times 10^{-6}$ & $4.42 \times 10^{-2}$ & $9.67 \times 10^{-3}$ & $1.49 \times 10^{-6}$ & $3.06 \times 10^{-3}$ & $1.97 \times 10^{-5}$ & $1.04 \times 10^{-4}$ & $6.75 \times 10^{-6}$ & $1.10 \times 10^{-5}$ & $4.32 \times 10^{-3}$ & $3.59 \times 10^{-3}$ & $8.31 \times 10^{-5}$ \\
\hline HQinh & $9.64 \times 10^{-8}$ & $5.92 \times 10^{-5}$ & $9.47 \times 10^{-6}$ & $4.08 \times 10^{-4}$ & $6.29 \times 10^{-4}$ & $5.77 \times 10^{-7}$ & $7.37 \times 10^{-7}$ & $2.54 \times 10^{-7}$ & $2.83 \times 10^{-7}$ & $6.32 \times 10^{-5}$ & $7.03 \times 10^{-6}$ & $1.63 \times 10^{-6}$ \\
\hline HQvaour & & & & & & & $1.04 \times 10^{-1}$ & & & & & \\
\hline $\mathrm{HI}=\sum \mathrm{HQ}$ & $6.61 \times 10^{-4}$ & $4.47 \times 10^{-1}$ & $7.40 \times 10^{-2}$ & $1.20 \times 10^{-3}$ & $4.45 \times 10^{-2}$ & $3.96 \times 10^{-3}$ & $1.09 \times 10^{-1}$ & $1.72 \times 10^{-3}$ & $1.99 \times 10^{-3}$ & $4.36 \times 10^{-1}$ & $5.14 \times 10^{-2}$ & $1.12 \times 10^{-2}$ \\
\hline \multicolumn{13}{|l|}{ Child } \\
\hline Ding & $2.38 \times 10^{-5}$ & $8.79 \times 10^{-4}$ & $4.68 \times 10^{-4}$ & $1.15 \times 10^{-4}$ & $8.90 \times 10^{-4}$ & $1.15 \times 10^{-3}$ & $1.06 \times 10^{-5}$ & $6.21 \times 10^{-5}$ & $2.88 \times 10^{-4}$ & $1.10 \times 10^{-2}$ & $1.39 \times 10^{-4}$ & $2.41 \times 10^{-2}$ \\
\hline $\mathrm{Dd} \times 10 \mathrm{rmal}$ & $2.74 \times 10^{-8}$ & $3.03 \times 10^{-5}$ & $5.38 \times 10^{-7}$ & $1.33 \times 10^{-7}$ & $1.02 \times 10^{-6}$ & $1.32 \times 10^{-6}$ & $1.22 \times 10^{-8}$ & $7.14 \times 10^{-8}$ & $3.31 \times 10^{-7}$ & $1.26 \times 10^{-5}$ & $1.60 \times 10^{-7}$ & $2.78 \times 10^{-5}$ \\
\hline Dinh & $6.66 \times 10^{-10}$ & $2.46 \times 10^{-8}$ & $1.31 \times 10^{-8}$ & $3.22 \times 10^{-9}$ & $2.49 \times 10^{-8}$ & $3.20 \times 10^{-8}$ & $2.95 \times 10^{-10}$ & $1.74 \times 10^{-9}$ & $8.05 \times 10^{-9}$ & $3.07 \times 10^{-7}$ & $3.88 \times 10^{-9}$ & $6.75 \times 10^{-7}$ \\
\hline Dvapour & & & & & & & $1.23 \times 10^{-5}$ & & & & & \\
\hline HQing & $4.76 \times 10^{-3}$ & $2.93 \times 10$ & $4.68 \times 10^{-1}$ & $5.76 \times 10^{-3}$ & $2.97 \times 10^{-1}$ & $2.87 \times 10^{-2}$ & $3.52 \times 10^{-2}$ & $1.24 \times 10^{-2}$ & $1.44 \times 10^{-2}$ & $3.14 \times 10$ & $3.47 \times 10^{-1}$ & $8.05 \times 10^{-2}$ \\
\hline $\mathrm{HQd} \times 10 \mathrm{rmal}$ & $3.04 \times 10^{-5}$ & $2.46 \times 10^{-1}$ & $5.38 \times 10^{-2}$ & $8.29 \times 10^{-6}$ & $1.71 \times 10^{-2}$ & $1.10 \times 10^{-4}$ & $5.79 \times 10^{-4}$ & $3.76 \times 10^{-5}$ & $6.14 \times 10^{-5}$ & $2.41 \times 10^{-2}$ & $2.00 \times 10^{-2}$ & $4.63 \times 10^{-4}$ \\
\hline HQinh & $1.33 \times 10^{-7}$ & $8.18 \times 10^{-5}$ & $1.31 \times 10^{-5}$ & $5.64 \times 10^{-4}$ & $8.70 \times 10^{-4}$ & $7.97 \times 10^{-7}$ & $1.02 \times 10^{-6}$ & $3.51 \times 10^{-7}$ & $3.91 \times 10^{-7}$ & $8.73 \times 10^{-5}$ & $9.71 \times 10^{-6}$ & $2.25 \times 10^{-6}$ \\
\hline HQvaour & & & $502 \times 10^{-1}$ & (1) & $15 \times 10-1$ & $200 \times 10-2$ & $1.43 \times 10^{-1}$ & $125 \times 10^{-2}$ & $145 \times 10^{-2}$ & & $367 \times 10^{-1}$ & $809 \times 10^{-2}$ \\
\hline $\mathrm{HI}=\sum \mathrm{HQ}$ & $4.80 \times 10^{-3}$ & $3.18 \times 10$ & $5.22 \times 10^{-1}$ & $6.34 \times 1$ & $3.15 \times 10^{-1}$ & $2.88 \times 10^{-2}$ & $1.79 \times 10^{-1}$ & $1.25 \times 10^{-2}$ & $1.45 \times 10^{-2}$ & $3.17 \times 10$ & $3.67 \times 10^{-1}$ & $8.09 \times 10^{-2}$ \\
\hline
\end{tabular}


Concerning the two population groups, they both experience potential health risk by $\mathrm{Pb}$ and $\mathrm{As}$ exposure from street dust. For children, the Hazard Indexes for $\mathrm{Pb}$ and As exceed the safe level (both greater than 3 , Table 4). There has a great possibility to pose potential adverse health effects to human beings that non-carcinogenic effects may occur, with a probability which tends to increase as the value of $\mathrm{HI}$ increases [15,16,24]. According to De Miguel et al. [31], adverse health effects may occur at HI values $>0.1$ level in the child cohort. Consequently, $\mathrm{Cd}, \mathrm{Cr}, \mathrm{Hg}$ and $\mathrm{Sb}$, that with HIs greater than 0.1 for the child, exposure to the street dust in Zhuzhou cannot be overlooked, and its ecological and health implications need investigated in detail in the further.

\subsection{Risks Associated with the Carcinogenic Elements As, Cd, Cr, Co and Ni}

The overall carcinogenic risks owing to exposure to $\mathrm{As}, \mathrm{Cd}, \mathrm{Co}, \mathrm{Cr}$ and $\mathrm{Ni}$ in street dust are shown in Table 5. The carcinogenic risk levels of $\mathrm{Cd}, \mathrm{Co}$ and Ni exposure for both cohorts are within the same low order of magnitude $\left(10^{-8} \sim 10^{-9}\right)$, which means that they pose a negligible risk [32,33].

In terms of children, the calculated value for $\mathrm{As}\left(1.04 \times 10^{-6}\right)$ and $\mathrm{Cr}\left(1.07 \times 10^{-6}\right)$ in street dust falls within the range of threshold values $\left(10^{-4}-10^{-6}\right)$ which is an internationally accepted precautionary risk criterion [32,33]. In contrast, adult exposure carcinogenic risk for these elements falls below this threshold $\left(1.29 \times 10^{-7}\right.$ and $6.05 \times 10^{-7}$ for As and $\mathrm{Cr}$, respectively). As in street dust is introduced by the nonferrous smelting activities and $\mathrm{Cr}$ is naturally enriched in the local soil of Zhuzhou [21]. As a carcinogen, arsenic can cause lung and skin cancer [34] and $\mathrm{Cr}$ can trigger lung cancer and stomach cancer [34] if an excessive buildup of both elements occurs. In view of the above, the risk of lung cancer disease increased by one times for the two subpopulations. Hence, further research should be undertaken to reveal the synergistic influence from $\mathrm{Cr}$ and As on human health.

Table 5. Daily exposure amounts and Cancer Risk of metals in street dust to adult and child through three routes.

\begin{tabular}{|c|c|c|c|c|c|}
\hline $\mathrm{mg} \cdot \mathrm{kg}^{-1} \cdot \mathrm{day}^{-1}$ & As & $\mathrm{Cd}$ & Co & $\mathrm{Cr}$ & $\mathbf{N i}$ \\
\hline \multicolumn{6}{|l|}{ Adult } \\
\hline LADD-Oral & $8.88 \times 10^{-8}$ & & & & \\
\hline LADD-Dermal & $4.00 \times 10^{-9}$ & & & & \\
\hline LADD-Inhal. & $1.78 \times 10^{-8}$ & $9.47 \times 10^{-9}$ & $2.33 \times 10^{-9}$ & $1.80 \times 10^{-8}$ & $5.83 \times 10^{-9}$ \\
\hline Risk-Oral & $1.33 \times 10^{-7}$ & & & & \\
\hline Risk-Dermal & $1.46 \times 10^{-8}$ & & & & \\
\hline Risk-Inhal & $2.68 \times 10^{-8}$ & $5.96 \times 10^{-8}$ & $2.28 \times 10^{-8}$ & $7.56 \times 10^{-7}$ & $4.90 \times 10^{-9}$ \\
\hline Risk-Total & $1.75 \times 10^{-7}$ & $5.96 \times 10^{-8}$ & $2.28 \times 10^{-8}$ & $7.56 \times 10^{-7}$ & $4.90 \times 10^{-9}$ \\
\hline \multicolumn{6}{|l|}{ Child } \\
\hline LADD-Oral & $6.46 \times 10^{-7}$ & & & & \\
\hline LADD-Dermal & $2.23 \times 10^{-8}$ & & & & \\
\hline LADD-Inhal. & $2.46 \times 10^{-8}$ & $1.31 \times 10^{-8}$ & $3.22 \times 10^{-9}$ & $2.49 \times 10^{-8}$ & $8.05 \times 10^{-9}$ \\
\hline Risk-Oral & $9.69 \times 10^{-7}$ & & & & \\
\hline Risk-Dermal & $8.16 \times 10^{-8}$ & & & & \\
\hline Risk-Inhal & $3.71 \times 10^{-8}$ & $8.24 \times 10^{-8}$ & $3.16 \times 10^{-8}$ & $1.04 \times 10^{-6}$ & $6.76 \times 10^{-9}$ \\
\hline Risk-Total & $1.09 \times 10^{-6}$ & $8.24 \times 10^{-8}$ & $3.16 \times 10^{-8}$ & $1.04 \times 10^{-6}$ & $6.76 \times 10^{-9}$ \\
\hline
\end{tabular}

\subsection{Comparison of HI and Cancer Risk with Other Researches}

The HI and cancer risk assessment results in street dust of Zhuzhou were compared with results from other cities around the world, as shown in Tables 6 and 7. For children, there is only one additional study (Huludao, Liaoning Province) that reported risk values of $\mathrm{Cd}$ and $\mathrm{Hg}$ exceeding 0.1. Huludao city once hosted the Asia's biggest zinc manufacturer. In addition, a study in Dongying city with large petrochemical industries reveal a $\mathrm{HI}$ larger than 0.1 for $\mathrm{Cr}$ [35], which was likewise found in this study. Similar discovery were obtained in municipal areas of Shanghai by Shi et al. [7] in a study of health risk of potentially metals in road dust. 
Table 6. Comparisons of hazard quotient for trace metals in urban dust of different cities.

\begin{tabular}{|c|c|c|c|c|c|c|c|c|c|c|c|c|c|c|}
\hline City & Factor & $\mathrm{Ag}$ & As & $\mathrm{Cd}$ & Co & $\mathrm{Cr}$ & $\mathrm{Cu}$ & $\mathrm{Hg}$ & Mo & $\mathrm{Ni}$ & $\mathrm{Pb}$ & $\mathrm{sb}$ & $\mathrm{Zn}$ & Literature \\
\hline \multicolumn{15}{|l|}{ Adult } \\
\hline $\begin{array}{l}\text { Zhuzhou, China } \\
\text { Huludao, China } \\
\text { Dongying, China }\end{array}$ & $\begin{array}{l}\text { Industrial pollution } \\
\text { Zinc smelting } \\
\text { Oil industry }\end{array}$ & $6.61 \times 10^{-4}$ & $\begin{array}{l}4.47 \times 10^{-1} \\
6.73 \times 10^{-2}\end{array}$ & $\begin{array}{l}7.40 \times 10^{-2} \\
2.58 \times 10^{-1} \\
2.46 \times 10^{-3}\end{array}$ & $\begin{array}{l}1.20 \times 10^{-3} \\
7.46 \times 10^{-4}\end{array}$ & $\begin{array}{l}4.45 \times 10^{-2} \\
2.24 \times 10^{-2}\end{array}$ & $\begin{array}{l}3.96 \times 10^{-3} \\
5.71 \times 10^{-3} \\
1.85 \times 10^{-3}\end{array}$ & $\begin{array}{l}1.09 \times 10^{-1} \\
8.79 \times 10^{-2}\end{array}$ & $1.72 \times 10^{-3}$ & $\begin{array}{l}1.99 \times 10^{-3} \\
1.86 \times 10^{-3}\end{array}$ & $\begin{array}{l}4.36 \times 10^{-1} \\
1.44 \times 10^{-1} \\
4.21 \times 10^{-2}\end{array}$ & $5.14 \times 10^{-2}$ & $\begin{array}{l}1.12 \times 10^{-2} \\
1.40 \times 10^{-2} \\
2.23 \times 10^{-3}\end{array}$ & $\begin{array}{l}\text { This study } \\
\text { Zheng et al. } 2010[15] \\
\text { Kong et al. } 2011[17]\end{array}$ \\
\hline $\begin{array}{l}\text { Zhuzhou, China } \\
\text { Huludao, China } \\
\text { Dongying, China }\end{array}$ & $\begin{array}{l}\text { Industrial pollution } \\
\text { Zinc smelting } \\
\text { Oil industry }\end{array}$ & $4.80 \times 10^{-3}$ & $\begin{array}{c}3.18 \times 10 \\
5.86 \times 10^{-1}\end{array}$ & $\begin{array}{l}5.22 \times 10^{-1} \\
6.16 \times 10^{-1} \\
2.10 \times 10^{-2}\end{array}$ & $\begin{array}{l}6.34 \times 10^{-3} \\
5.04 \times 10^{-3}\end{array}$ & $\begin{array}{l}3.15 \times 10^{-1} \\
1.96 \times 10^{-1}\end{array}$ & $\begin{array}{l}2.88 \times 10^{-2} \\
4.75 \times 10^{-2} \\
1.73 \times 10^{-2}\end{array}$ & $\begin{array}{l}1.79 \times 10^{-1} \\
1.82 \times 10^{-1}\end{array}$ & $1.25 \times 10^{-2}$ & $1.45 \times 10^{-2}$ & $\begin{array}{c}3.17 \times 10 \\
1.08 \times 10 \\
3.90 \times 10^{-1}\end{array}$ & $3.67 \times 10^{-1}$ & $\begin{array}{l}8.09 \times 10^{-2} \\
1.11 \times 10^{-1} \\
2.07 \times 10^{-2}\end{array}$ & $\begin{array}{l}\text { This study } \\
\text { Zheng et al. 2010 [15] } \\
\text { Kong et al. 2011 [17] }\end{array}$ \\
\hline Luanda, Angola & Industrial pollution & $8.42 \times 10^{-4}$ & $1.36 \times 10^{-1}$ & $1.01 \times 10^{-2}$ & $1.11 \times 10^{-3}$ & $6.63 \times 10^{-2}$ & $7.30 \times 10^{-3}$ & $1.61 \times 10^{-2}$ & $2.81 \times 10^{-3}$ & $3.55 \times 10^{-3}$ & $7.23 \times 10^{-1}$ & $6.58 \times 10^{-2}$ & $7.40 \times 10^{-3}$ & $\begin{array}{l}\text { Ferreira-Baptista and } \\
\text { De Miguel, } 2005 \text { [14] }\end{array}$ \\
\hline Madrid, Spain & Industrial pollution & $1.67 \times 10^{-4}$ & $1.09 \times 10^{-1}$ & $1.09 \times 10^{-3}$ & & $2.72 \times 10^{-2}$ & $1.41 \times 10^{-3}$ & $1.71 \times 10^{-2}$ & $6.00 \times 10^{-4}$ & $9.61 \times 10^{-4}$ & $3.11 \times 10^{-2}$ & $1.06 \times 10^{-2}$ & $7.10 \times 10^{-4}$ & De Miguel et al. 2007 [31] \\
\hline
\end{tabular}

Table 7. Comparisons of cancer risk for child in urban dust of different cities.

\begin{tabular}{|c|c|c|c|c|c|c|c|}
\hline City & Factor & As & Cd & Co & $\mathrm{Cr}$ & $\mathrm{Ni}$ & Literature \\
\hline Zhuzhou, China & Industrial pollution & $1.04 \times 10^{-6}$ & $8.46 \times 10^{-8}$ & $3.24 \times 10^{-8}$ & $1.07 \times 10^{-6}$ & $6.94 \times 10^{-9}$ & This study \\
\hline Huludao, China & Zinc smelting & & & & $2.36 \times 10^{-8}$ & & Zheng et al. 2010 [15] \\
\hline Dongying, China & Oil industry & $7.21 \times 10^{-7}$ & $8.06 \times 10^{-10}$ & $4.35 \times 10^{-8}$ & & $2.94 \times 10^{-8}$ & Kong et al. 2011 [17] \\
\hline Luanda, Angola & Industrial pollution & $7.69 \times 10^{-6}$ & $3.89 \times 10^{-10}$ & $1.53 \times 10^{-9}$ & $5.70 \times 10^{-8}$ & $4.61 \times 10^{-10}$ & Ferreira-Baptista and De Miguel, 2005 [14] \\
\hline Madrid, Spain & Industrial pollution & $4.19 \times 10^{-6}$ & $2.62 \times 10^{-11}$ & & $1.73 \times 10^{-8}$ & $1.37 \times 10^{-9}$ & De Miguel et al. 2007 [31] \\
\hline
\end{tabular}


Furthermore, hazard indexes of As and $\mathrm{Pb}$ for children exposure with value higher $10^{-1}$ appeared in several studies (see Table 6), so, these two elements could trigger neurological and developmental disorders [14]. As the same time, arsenic was the only element that associated with carcinogenic risk exceeding $10^{-6}$ in the reviewed studies. As and $\mathrm{Pb}$ appear to have a widespread impact on children health in polluted cities all over the world.

Since arsenic is outstanding both for cancer and non-cancer risk for many researches. Here we take arsenic as an example, compared the results obtained by this study and other researches. The HI and cancer risk of As for children are calculated according to the date from other literatures [33,36-39]. The HI means of these cities are less than 0.1 (Figure 2) and the cancer risk means are in $10^{-7}$ to $10^{-8}$ level (Figure 2). The maximum of $\mathrm{HI}$ in Wuhu (China) and Singapore are $9.29 \times 10^{-1}, 9.66 \times 10^{-1}$, respectively, which are very close to one. While the data indicated that the $\mathrm{HI}$ and cancer risk of As in Zhuzhou are much serious than other cities $\left(\mathrm{HI}>3\right.$, and cancer risk $\left.>10^{-6}\right)$, indicating a more worrying polluted status.

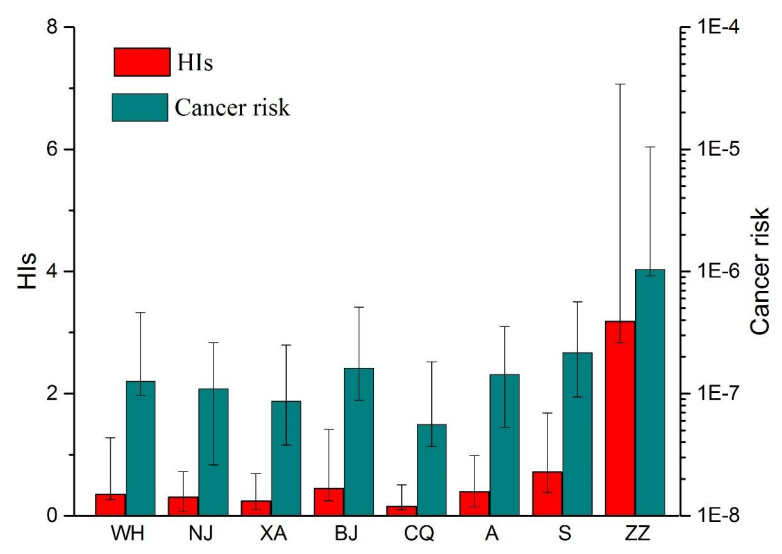

Figure 2. A comparison of HIs and cancer risk of arsenic to children in street dust of Wuhu (WH), Nanjing (NJ), Xi'an (XA), Baoji (BJ), Chongqing (CQ), Avilés (A), Singapore (S) and this study (ZZ).

Meanwhile, for adults, HIs for Ag, As, Cd, Co, Cr, Hg, Sb and $\mathrm{Zn}$ in these cities (Zhuzhou, Huludao and Dongying are lower than 1, suggesting that there is little adverse health risk due to street dust. From the aforementioned statements, it could be concluded that As and $\mathrm{Pb}$ contamination has become a serious environmental problem in a category of cities and further research should be undertaken to explore the particular influence of As on children health.

\section{Conclusions}

This study elucidates that children's health is specifically at risk when exposed to street dust enriched in toxic metals and metalloids such as $\mathrm{Pb}$ and As, as the case study in Zhuzhou city of Hunan Province, China, showed. Hazard quotient calculations indicate that ingestion of dust particles pose the highest risk to children and adults in Zhuzhou, followed by dermal contact and subsequently inhalation for all the metals included except $\mathrm{Hg}$, for which inhalation of its vapor presents a slightly higher risk than ingestion. For adults, risk values of both carcinogenic and non-carcinogenic health effects in this study were in the receivable range. For children, $\mathrm{Pb}, \mathrm{As}, \mathrm{Cd}, \mathrm{Cr}, \mathrm{Hg}$ and $\mathrm{Sb}$ are regarded as the most possible contributor to non-carcinogenic health risks, while As and $\mathrm{Cr}$ increase the risk for causing cancer. Compared the HI and cancer risk of arsenic for children in this study to others, we found arsenic was regarded as the most possible element to cancer and non-cancer risks for children in those cities (Huludao, Dongying, Luanda, Madrid). Hence, further study should pay more attention to the effects of As in dust on children's health.

Acknowledgments: The authors would like to acknowledge the support of the National Key Basic Research Program of China (No. 2013CB430001) and the Natural Science Foundation of China (No.41373056). 
Author Contributions: Zhonggen Li and Xinbin Feng conceived and designed the experiments; Guangyi Sun Ting Liu, Ji Chen and Tingting Wu performed the experiments; Guangyi Sun and Zhonggen Li analyzed the data; Zhonggen Li contributed reagents/materials/analysis tools; Guangyi Sun wrote the paper. All authors read and approved the final manuscript.

Conflicts of Interest: The authors declare no conflict of interest.

\section{References}

1. Banerjee, A.D.K. Heavy metal levels and solid phase speciation in street dusts of Delhi, India. Environ. Pollut. 2003, 123, 95-105. [CrossRef]

2. Zhang, X.M.; Luo, K.L.; Sun, X.Z.; Tan, J.A.; Lu, Y.L. Mercury in the topsoil and dust of Beijing City. Sci. Total Environ. 2006, 368, 713-722.

3. Manno, M.; Varrica, D.; Dongarra, G. Metal distribution in road dust samples collected in an urban area close to a petrochemical plant at Gela, Sicily. Atmos. Environ. 2006, 40, 5929-5941. [CrossRef]

4. Zhang, M.K.; Wang, H. Concentrations and chemical forms of potentially toxic metals in road-deposited sediments from different zones of Hangzhou, China. J. Environ. Sci. 2009, 21, 625-631. [CrossRef]

5. Lu, X.W.; Wang, L.J.; Lei, K.; Huang, J.; Zhai, Y.X. Contamination assessment of copper, lead, zinc, manganese and nickel in street dust of Baoji, NW China. J. Hazard. Mater. 2009, 161, 1058-1062. [CrossRef] [PubMed]

6. Bi, X.Y.; Li, Z.G.; Zhuang, X.C.; Han, Z.X.; Yang, W.L. High levels of antimony in dust from e-waste recycling in southeastern China. Sci. Total Environ. 2011, 409, 5126-5128. [CrossRef] [PubMed]

7. Shi, G.T.; Chen, Z.L.; Bi, C.J.; Wang, L.; Teng, J.Y.; Li, Y.S.; Xu, S.Y. A comparative study of health risk of potentially toxic metals in urban and suburban road dust in the most populated city of China. Atmos. Environ. 2011, 45, 764-771. [CrossRef]

8. Shi, G.T.; Chen, Z.L.; Xu, S.Y.; Zhang, J.; Wang, L.; Bi, C.J.; Teng, J.Y. Potentially toxic metal contamination of urban soils and roadside dust in Shanghai, China. Environ. Pollut. 2008, 156, 251-260. [CrossRef] [PubMed]

9. Rico, D.; Martin-González, A.; Diaz, S.; De Lucas, P.; Gutiérrez, J.C. Heavy metals generate reactive oxygen species in terrestrial and aquatic ciliated protozoa. Comp. Biochem. Physiol. C Toxicol. Pharmacol. 2009, 149, 90-96. [CrossRef] [PubMed]

10. Godt, J.; Scheidig, F.; Grosse-Siestrup, C.; Esche, V.; Brandenburg, P.; Reich, A.; Groneberg, D.A. The toxicity of cadmium. J. Occup. Med. Toxicol. 2006, 22, 1-6.

11. Haefliger, P.; Mathieu-Nofl, M.; Lociciro, S.; Ndiaye, C.; Coly, M.; Diouf, A.; Faye, A.L.; Sow, A.; Tempowski, J.; Pronczuk, J.; et al. Mass lead intoxication from informal used lead-acid battery recycling in Dakar, Senegal. Environ. Health Perspect. 2009, 117, 1535-1540. [CrossRef] [PubMed]

12. Smith, A.H.; Marshall, G.; Yuan, Y.; Catterina, F.; Jane, L.; Ondine, V.E.; Craig, S.; Michael, N.B.; Steve, S. Increased mortality from lung cancer and bronchiectasis in young adults after exposure to arsenic in utero and in early childhood. Environ. Health Perspect. 2006, 114, 1293-1296. [CrossRef] [PubMed]

13. Sun, G.Y.; Li, Z.G.; Bi, X.Y.; Chen, Y.P.; Lu, S.F.; Yuan, X. Distribution, sources and health risk assessment of mercury in kindergarten dust. Atmos. Environ. 2013, 73, 169-176. [CrossRef]

14. Ferreira-Baptista, L.; De Miguel, E. Gechemistry and risk assessment of street dust in Luanda, Angola: A tropical urban environment. Atmos. Environ. 2005, 38, 4501-4512. [CrossRef]

15. Zheng, N.; Liu, J.S.; Wang, Q.C.; Liang, Z.Z. Health risk assessment of heavy metal exposure to street dust in the zinc smelting district, Northeast of China. Sci. Total Environ. 2010, 408, 726-733. [CrossRef] [PubMed]

16. Zheng, N.; Liu, J.S.; Wang, Q.C.; Liang, Z.Z. Heavy metals exposure of children from stairway and sidewalk dust in the smelting district, northeast of China. Atmos. Environ. 2010, 44, 3239-3245. [CrossRef]

17. Kong, S.F.; Lu, B.; Bai, Z.P.; Zhao, X.Y.; Chen, L.; Han, B.; Li, Z.Y.; Ji, Y.Q.; Xu, Y.H.; Liu, Y.; et al. Potential threat of heavy metals in re-suspended dusts on building surfaces in oilfield city. Atmos. Environ. 2011, 45, 4192-4204. [CrossRef]

18. Wang, W.; Huang, M.J.; Kang, Y.; Wang, H.S.; Leung, A.O.W.; Cheung, K.C.; Wong, M.H. Polycyclic aromatic hydrocarbons (PAHs) in urban surface dust of Guangzhou, China: Status sources and human health risk assessment. Sci. Total Environ. 2011, 409, 4519-4527. [CrossRef] [PubMed]

19. Faiz, Y.; Siddioue, N.; Tufail, M. Pollution level and health risk assessment of road dust from an expressway. J. Environ. Sci. Health 2012, 47, 818-829. [CrossRef] [PubMed] 
20. U.S. DOE (United States Department of Energy). Available online: http://science.energy.gov/ / media/budget/pdf/sc-budget-request-to-congress/fy-2004/Cong_Budget_2004_BER.pdf (accessed on 10 December 2016).

21. Li, Z.G.; Feng, X.B.; Li, G.H.; Bi, X.Y.; Zhu, J.M.; Qiu, H.B.; Dai, Z.H.; Liu, J.L.; Li, Q.H.; Sun, G.Y. Distribution, sources and pollution status of 17 trace metal/metalloids in the street dust of a heavily industrialized city of central China. Environ. Pollut. 2013, 182, 408-416. [CrossRef] [PubMed]

22. U.S. EPA (United States Environmental Protection Agency). Human Health Evaluation Manual. EPA/540/1-89/002. Risk Assessment Guidance for Superfund; Office of Soild Waste and Emergency Response: Washington, DC, USA, 1989.

23. U.S. EPA (United States Environmental Protection Agency). Soil Screening Guidance: Technical Background Document; EPA/540/R-95/128; Office of Soild Waste and Emergency Response: Washington, DC, USA, 1996.

24. U.S. EPA (United States Environmental Protection Agency). Supplemental Guidance for Developing Soil Screening Levels for Superfund Sites; OSWER 9355.4-24; Office of Solid Waste and Emergency Response: Washington, DC, USA, 2001.

25. Naturvårdsverket, S. Development of Generic Guideline Values. Model and Data Used for Generic Guideline Values for Contaminated Soils in Sweden; Report 4639; Swedish Environmental Protection Agency: Stockholm, Sweden, 1996.

26. U.S. EPA (United States Environmental Protection Agency). Guidance for Evaluating the Oral Bioavailability of Metals in Soils for Use in Human Health Risk Assessment; OSWER 9285.7-80; EPA: Washington, DC, USA, 2007.

27. Van den Berg, R. Human Exposure to Soil Contamination: A Qualitative and Quantitative Analysis towards Proposals for Human Toxicological Intervention Values; RIVM Report No. 725201011; National Institute of Public Health and Environmental Protection (RIVM): Bilthoven, The Netherlands, 1995.

28. Wang, Z.; Liu, S.; Chen, X.; Lin, C. Estimates of the exposed dermal surface area of Chinese in view of human health risk assessment. J. Saf. Environ. 2008, 4, 152-156. (In Chinese)

29. Wang, X.L.; Sato, T.; Xing, B.S.; Tao, S. Health risks of heavy metals to the general public in Tianjin, China via consumption of vegetables and fish. Sci. Total Environ. 2005, 350, 28-37. [CrossRef] [PubMed]

30. CNEMC (China National Environmental Monitoring Centre). The Background Values of Chinese Soils; Environmental Science Press: Beijing, China, 1990. (In Chinese)

31. De Miguel, E.; Iribarren, I.; Chacón, E.; Ordoñez, A.; Charlesworth, S. Risk-based evaluation of the exposure of children to trace elements in playgrounds in Madrid (Spain). Chemosphere 2007, 66, 505-513. [CrossRef] [PubMed]

32. Meza-Figueroa, D.; De la O-Villanueva, M.; De la Parra, M.L. Heavy metal distribution in dust from elementary schools in Hermosillo, Sonora, Mexico. Atmos. Environ. 2007, 41, 276-288. [CrossRef]

33. Hu, X.; Zhang, Y.; Luo, J.; Wang, T.J.; Lian, H.; Ding, Z. Bioaccessibility and health risk of arsenic, mercury and other metals in urban street dusts from a mega-city, Nanjing, China. Environ. Pollut. 2011, 159, 1215-1221. [CrossRef] [PubMed]

34. U.S. EPA (United States Environmental Protection Agency). Integrated Risk Information System (IRIS), 2005. Available online: http://www.epa.gov/iris/subst/0278.htm\#carc (accessed on 10 December 2016).

35. Kong, S.F.; Lu, B.; Ji, Y.Q.; Zhao, X.Y.; Bai, Z.P.; Xu, Y.H.; Liu, Y.; Jiang, H. Risk assessment of heavy metals in road and soil dusts within $\mathrm{PM}_{2.5}, \mathrm{PM}_{10}$ and $\mathrm{PM}_{100}$ fractions in Dongying city, Shangdong Province, China. J. Environ. Monit. 2012, 14, 791-803. [CrossRef] [PubMed]

36. Batjargal, T.; Otgonjargal, E.; Baek, K.; Yang, J.S. Assessment of metals contamination of soils in Ulaanbaatar, Mongolia. J. Hazard. Mater. 2010, 184, 872-876. [CrossRef] [PubMed]

37. Joshi, U.M.; Vijayaraghavan, K.; Balasubramanian, R. Elemental composition of urban street dusts and their dissolution characteristics in various aqueous media. Chemosphere 2009, 77, 526-533. [CrossRef] [PubMed]

38. Wang, X.L. Pollution Analyse of Street Dust in Core District of Chongqing. Master Dissertation, Southwest University, Chongqing, China, 2008; p. 20. (In Chinese)

39. Fang, F.M.; Zhang, Z.M.; Chen, W.J.; Yang, D. Spatial and particle size distribution of mercury and arsenic on surface dust in spring in the Wuhu urban district. Acta Sci. Circumst. 2009, 29, 1871-1877. (In Chinese)

(C) 2017 by the authors. Licensee MDPI, Basel, Switzerland. This article is an open access article distributed under the terms and conditions of the Creative Commons Attribution (CC BY) license (http:/ / creativecommons.org/licenses/by/4.0/). 